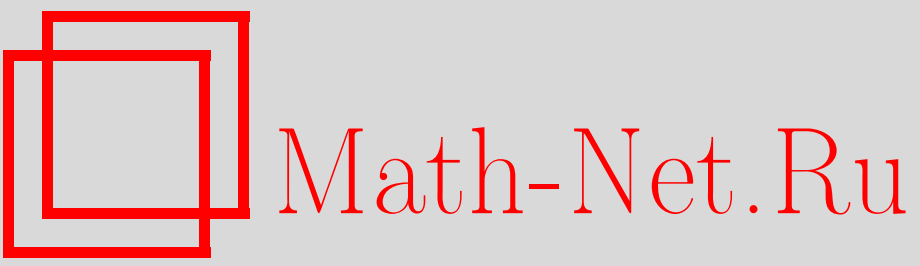

С. П. Суетин, О многочленах, ортогональных на нескольких отрезках с индефинитным весом, УМН, 2005, том 60, выпуск 5, 181-182

DOI: https://doi.org/10.4213/rm1655

Использование Общероссийского математического портала Math-Net.Ru подразумевает, что вы прочитали и согласны с пользовательским соглашением

http://www.mathnet.ru/rus/agreement

Параметры загрузки:

IP : 34.239 .49 .27

26 апреля 2023 г., $11: 57: 11$ 


\title{
О МНОГОЧЛЕНАХ, ОРТОГОНАЛЬНЫХ НА НЕСКОЛЬКИХ ОТРЕЗКАХ С ИНДЕФИНИТНЫМ ВЕСОМ
}

\author{
С. П. Суетин
}

1. Как известно [1], развитый П. Л. Чебьшёвым метод решения ряда экстремальных задач с помощью аппарата непрерывных дробей привел его [2] к открытию общих ортогональных многочленов, которые возникли как знаменатели $Q_{n}$ подходящих дробей $P_{n} / Q_{n}, \operatorname{deg} P_{n} \leqslant n$, $\operatorname{deg} Q_{n} \leqslant n, n=1,2, \ldots$, к непрерывной $J$-дроби, ассоциированной с функцией вида

$$
\widehat{\mu}(z)=\int_{S} \frac{d \mu(x)}{z-x}, \quad z \in \overline{\mathbb{C}} \backslash S,
$$

где $\mu$-положительная борелевская мера с носителем $S \Subset \mathbb{R}$. Вопрос о сходимости такой $J$-дроби (т.е. последовательности $\left\{P_{n} / Q_{n}\right\}_{n \in \mathbb{N}}$ ) был изучен А.А. Марковым [3], которьй доказал, что $J$-дробь равномерно сходится к $\widehat{\mu}$ вне отрезка $[\alpha, \beta]$ - выпуклой оболочки $S$.

Пусть $\mu>0$, носитель $S$ состоит из несколких отрезков: $S=\Delta_{1} \sqcup \cdots \sqcup \Delta_{p}, p \geqslant 2$, и

$$
d \mu(x)=\frac{\rho(x) d x}{\sqrt{-h(x+i 0)}}, \quad \text { где функция } \rho \neq 0 \text { и голоморффна на } S,
$$

$h(z)=\prod_{j=1}^{p}\left(z-\alpha_{j}\right)\left(z-\beta_{j}\right), \Delta_{j}=\left[\alpha_{j}, \beta_{j}\right]$, и в $\overline{\mathbb{C}} \backslash S$ выбрана такая ветвь корня, что $\sqrt{h(z)} \sim z^{p}$ при $z \rightarrow \infty$. В "типичном" случае $\overline{\mathbb{C}} \backslash[\alpha, \beta]$ - максимальная область равномерной сходимости последовательности $\left\{P_{n} / Q_{n}\right\}_{n \in \mathbb{N}}$. Точнее, справедлива [4]-[7] следующая

Теорема. Если гармонические меры $\omega_{1}(\infty), \ldots, \omega_{p}(\infty)$ отрезков $\Delta_{1}, \ldots, \Delta_{p}$ рачионально независимы, то нули ортогональных многочленов $\left\{Q_{n}\right\}_{n \in \mathbb{N}}$ всюду плотны в лакунах $\left[\beta_{j}, \alpha_{j+1}\right], j=1, \ldots, p-1$, между отрезками.

2. Пусть $f=\sum_{k=0}^{\infty} c_{k} / z^{k+1}$ - степенной ряд, $\left\{[n / n]_{f}\right\}_{n \in \mathbb{N}}-$ последовательность его диагональных аппроксимаций Паде: $[n / n]_{f}=P_{n} / Q_{n}$, где $\operatorname{deg} P_{n}, \operatorname{deg} Q_{n} \leqslant n, Q_{n} \not \equiv 0$ и $R_{n}(z):=$ $\left(Q_{n} f-P_{n}\right)(z)=O\left(1 / z^{n+1}\right)$ при $z \rightarrow \infty ; R_{n}-$ функция остатка. Для $f=\widehat{\mu}$ (см. (1)) функция $[n / n]_{\widehat{\mu}}$ - в точности $n$-я подходящая дробь к $J$-дроби, ассоциированной с $\widehat{\mu}$. Как известно [8], [9], сходимость $[n / n]_{f}$ фактически определяется поведением нулей $Q_{n}$.

Пусть $f=\widehat{\mu}$, мера $\mu$ задана (2), а $\rho$ - такая вещественнозначная функция, что $\mu$ может иметь на разных компонентах $S$ разные знаки, т.е. $\rho / \sqrt{-h}-$ индефинитный вес; в далњейшем будем называть весом функцию $\rho$. В [6] показано, что асимптотика тех нулей $Q_{n}$, которые имеют предельные точки вне $S$ (будем назьвать такие нули "ло жны ьми"; см. [8], [10]), при некоторых "начальных" условиях полностью описывается в терминах асимптотического поведения дивизора $d(n)=\mathbf{z}_{1}(n)+\cdots+\mathbf{z}_{g}(n)$, удовлетворяющего проблеме обращения Якоби с правой частью, зависящей от $\rho$ и индекса $n$. Точнее, пусть $\mathfrak{R}: w^{2}=h(z)$ - двулистная гиперэллиптическая риманова поверхность рода $g=p-1, \mathbf{z}=(z, w)$ - точки на $\mathfrak{R}$, где $w=\sqrt{h}$ на первом листе и $w=-\sqrt{h}-$ на втором; $d \Omega_{k}(\mathbf{z})=p_{k}(z) d z / w, k=1, \ldots, g,-$ стандартный $a$-нормированньй базис абелевых дифференциалов на $\mathfrak{R}, B_{k j}-b$-периоды этих дифференциалов, а $\Omega_{k}(\mathbf{z})$ - соответствующие многозначные абелевы функции на $\mathfrak{R}$. Пусть дивизор $d^{0}=\mathbf{z}_{1}^{0}+\cdots+\mathbf{z}_{g}^{0}$ удовлетворяет системе сравнений (проблеме обращения Якоби) $\sum_{j=1}^{g} \Omega_{k}\left(\mathbf{z}_{j}^{0}\right) \equiv \xi_{k}^{0}, k=1, \ldots, g$, где знак 三 означает равенство по модулю периодов базисных диффференциалов, а величины $\xi_{k}^{0}$ определяются весом $\rho$ [6]. Как известно [11], если дивизор $d^{0}$ - неспециальный, то он является единств еннылм решением этой системы и, тем самым, однозначно определяется $x, y$-координатами вектора $\xi^{0}=\left(\xi_{1}^{0}, \ldots, \xi_{g}^{0}\right)$ в многообразии Якоби Јас $\mathfrak{R}$ :

$$
x_{k}^{0}=-\frac{1}{\pi} \int_{S} \arg \rho(x) d \Omega_{k}^{+}(x)(\bmod 1), \quad y_{k}^{0}=-\frac{g}{2} \omega_{k}(\infty)+\rho_{k}(\bmod 1), \quad k=1, \ldots, g,
$$

Работа вьполнена при поддержке РФФИ (грант № 05-01-01027), фонда INTAS (грант № 0351-6637) и программы поддержки ведущих научных школ России (грант № НШ-1551.2003.1). 
где $d \Omega_{k}^{+}(x)=d \Omega_{k}(x+i 0), \sum_{k=1}^{g} \rho_{k} B_{k j}=\frac{i}{\pi} \int_{S} \log |\rho(x)| d \Omega_{j}^{+}(x), j=1, \ldots, g$ [6]. Пусть $d(n)=\mathbf{z}_{1}(n)+\cdots+\mathbf{z}_{g}(n)-$ последовательность дивизоров, удовлетворяющих при каждом $n=0,1,2, \ldots$ системе сравнений

$$
\sum_{j=1}^{g} \Omega_{k}\left(\mathbf{z}_{j}\right) \equiv \xi_{k}, \quad k=1, \ldots, g, \quad \text { где } \xi_{k}=x_{k}^{0}+\sum_{j=1}^{g} y_{j}^{0} B_{k j}+n \sum_{j=1}^{g} \omega_{j}(\infty) B_{k j} .
$$

Имеем: $x_{k}(n)=x_{k}^{0}, y_{k}(n)=y_{k}^{0}+n \omega_{k}(\infty)(\bmod 1)$, т.е. $x$-координаты не зависят от $n$. Если дивизор $d=\mathbf{z}_{1}+\cdots+\mathbf{z}_{g}$ таков, что $z_{j} \in\left[\alpha_{j}, \beta_{j}\right]$, то он неспециальный и имеет $x$-координаты $x_{k}=(g-k+1) / 2(\bmod 1) ;$ верно и обратное [6]. Пусть вес $\rho$ такой, что для $d(0)=d^{0}$ имеем: все $z_{j}^{0} \in\left[\alpha_{j}, \beta_{j}\right]$. Тогда и при каждом $n=1,2, \ldots$ все $z_{j}(n) \in\left[\alpha_{j}, \beta_{j}\right]$, дивизор $d(n)$ неспециальный, а динамика ложных нулей $Q_{n}$ полностью определяется динамикой $y$-координат $d(n)$. Отсюда уже с помощю развитого в [4]-[6] метода, основанного на исследовании специальной краевой задачи Римана на $\mathfrak{R}$ и сингулярного интегралшного уравнения Наттолла, вытекает сформулированная вьше теорема. Приведенные рассуждения остаются справедливыми и при замене одной из лакун $\left[\alpha_{j}, \beta_{j}\right]$ бесконечной лакуной, поэтому этот подход позволяет получить новые резултаты и для случая индефинитного веса $\rho$.

3. Пусть $S$ состоит из двух отрезков: $S=\Delta_{1} \sqcup \Delta_{2},[\alpha, \beta]$ - выпуклая оболочка $S$ и выполнено условие "общего положения": число $\omega_{1}(\infty)$ - иррационально (см. [12]).

Если $\rho / \sqrt{-h}$ меняет знак на $S$, то из свойства ортогональности $Q_{n}$ вытекает, что ложный нуль $Q_{n}$ может располагаться лишь во внешней лакуне $\overline{\mathbb{R}} \backslash[\alpha, \beta]$. Это вытекает и из приведенных выше рассуждений, основанных на (3)-(4). Действительно, так как $\arg \rho=0$ на $S$, то $x_{1}^{0}=0(\bmod 1)$. Такую же $x$-координату имеют все дивизоры $d(n)=\mathbf{z}_{1}(n)$. Следовательно, $z_{1}(n) \in \overline{\mathbb{R}} \backslash[\alpha, \beta]$ при всех $n \geqslant 0$.

ПредЛОЖенИЕ. Пусть в условиях общего положения $\rho / \sqrt{-h}$ меняет знак на $S$. Тогда $\mathbb{N}=\Lambda_{1} \sqcup \Lambda_{2} \sqcup \Lambda_{3}$, где плотности последовательностей $\Lambda_{1}=\Lambda_{1}(\rho)$ u $\Lambda_{2}=\Lambda_{2}(\rho)$ равны $1 / 2$ и

1) множество $\left\{z_{n}: n \in \Lambda_{1}, z_{n} \in \mathbb{R} \backslash[\alpha, \beta], Q_{n}\left(z_{n}\right)=0\right\}$ всюду плотно в $\overline{\mathbb{R}} \backslash(\alpha, \beta)$,

2) множество $\left\{\zeta_{n}: n \in \Lambda_{2}, \zeta_{n} \in \mathbb{R} \backslash[\alpha, \beta], R_{n}\left(\zeta_{n}\right)=0\right\}$ всюду плотно в $\overline{\mathbb{R}} \backslash(\alpha, \beta)$.

Аналогичные резултаты справедливы и для случая нескольких отрезков; исследование этого общего случая также основано на соотношениях (3)-(4).

\section{СПИСОК ЛИТЕРАТУРЫ}

[1] Н. И. Ахиезер // Математика ХІХ века / Под ред. А. Н. Колмогорова и А. П. Юшкевича. М.: Наука, 1987. С. 9-79. [2] П. Л. Чебышев. О непрерьвных дробях // Ученые записки Имп. Акад. Наук. 1855. Т. III. С. 636-664. [3] А. А. Марков. Deux démonstrations de la convergence de certaines fractions continues // Acta Math. 1895. V. 19. P. 93-104. [4] C. П. Суетин // Матем. сб. 2000. Т. 191. № 9. С. 81-114. [5] С. П. Суетин // УМН. 2002. Т. 57. № 1. С. 45-142. [6] С. П. Суетин // Матем. сб. 2002. Т. 193. № 12. С. 105-133. [7] F. Peherstorfer. Zeros of polynomials orthogonal on several intervals // arXiv: math-ph/0203058. [8] Дж. Бейкер, П. Грейвс-Моррис. Аппроксимации Паде. М.: Мир, 1986. [9] А. А. Гончар // Матем. сб. 1982. Т. 118. № 4. С. 535-556. [10] С. П. Суетин // УМН. 2004. Т. 59. № 4. С. 201-202. [11] Э. И. Зверович // УМН. 1971. Т. 26. № 1. С. 113-180. [12] С. П. Суетин // Матем. сб. 2003. T. 194. № 12. C. $63-92$.

Математический институт

им. В.А. Стеклова РАН

E-mail: suetin@mi.ras.ru
Представлено А. Г. Сергеевьм Принято редколлегией 07.09.2005 